\title{
Prevalence of Ceserean Section and Associated Factors in University of Gondar Comprehensive Referal Hospital, North West Ethiopia, 2019
}

Abayneh Aklilu Solomon ( $\sim$ sabayneh7@gmail.com )

\section{Research note}

Keywords: prevalence, Caesarean section, University of Gondar Referral Hospital, laboring mothers, Ethiopia.

Posted Date: August 21st, 2019

DOI: https://doi.org/10.21203/rs.2.13345/v1

License: (c) (i) This work is licensed under a Creative Commons Attribution 4.0 International License. Read Full License 


\section{Abstract}

Abstract Objective: cesarean section is the delivery of the fetus, placenta, and membranes through an incision on the abdominal and uterine walls after the fetus has reached viability. The world health organization (WHO) suggests the cesarean section (CS) rate between 5\%-15\%. Evidence suggested that the rate of CS is high in developing countries including Ethiopia. So the aim of this study was to assess the prevalence and associated factors of cesarean section in the University of Gondar comprehensive referral hospital, North West Ethiopia, 2019 Result: The overall prevalence of cesarean section in Gondar university hospital was $29.7 \%$. The most common indications of CS were non-reassurance fetal heart rate pattern (NRFHP) (17.8\%) which was followed by previous CS scar $(15.9 \%)$ and severe preeclampsia (12.1 \%). Factors such as maternal educational status (AOR 2.89, $\mathrm{Cl}$ (1.278-6.56), gravidity (AOR $3.259 \mathrm{Cl}$ (1.484-7.160), Ante Natal care (ANC) follow up (AOR 0.248, Cl (0.083-0.739) and number of ANC follow up (AOR $5.17 \mathrm{Cl}$ (1.48-18.03) were associated with CS. Keywords: prevalence, Caesarean section, University of Gondar Referral Hospital, laboring mothers, Ethiopia.

\section{Background}

Cesarean section(CS) is the delivery of the fetus, placenta, and membranes through an incision made on the mothers abdominal and uterine walls after 28th weeks of gestation(1). CS is the most common surgical procedure during pregnancy and labor to save both the life of the mother and the newborn (2). Despite its advantage, CS is associated with adverse maternal and neonatal outcomes including long term sequels, with appropriate clinical indication significantly saves the life of the mother and the newborn as well. In contrast, CS performed without any medical indication increases the trend without giving any advantage for the patients(3). Increased CS rate has an important negative implication for health coverage nationally and internationally (4).

Cesarean section performed with aseptic technique, appropriate anesthesia, the applicability of lower transverse uterine segment cesarean section, safe and rapid availability of blood products collectively decreases the morbidity and mortality associated with cesarean section(5). The rate of cesarean section is twice higher in private than in public hospitals $(6,7)$.

The global cesarean section rate (CSR) is $18.6 \%$ ranging between $6.0 \%$ to $27.2 \%$ in developing and developed world respectively, Latin American and Caribbean accounts the highest proportion of cesarean section rate (40.5\%) and the lowest rate is in Africa (7.3\%), particularly in western Africa (3\%)(2).

The major obstetrical indication for cesarean section is obstructed labor, previous CS scar, non-reassuring fetal heart pattern, malpresentation, malposition, antepartum hemorrhage and failed induction, cesarean section rate more than the WHO threshold (15\%) cause morbidity and mortality than giving any advantage, so routine use of antibiotics for all women undergoing CS decreases the morbidities associated with $\operatorname{CS}(8,9)$. 
According to the WHO 2010 report, the minimum CS rate to have a good maternal outcome is $5 \%$. However, for better neonatal health outcome the range should be between $5 \%-10 \%(4)$

Studies conducted in three Asian countries the prevalence of CS in Bangladesh was 73\%, 30\% in Nepal and $18 \%$ in India(7). Studies also that was done in brazil was $29.9 \%$ and $86.2 \%$ in public and private health sectors respectively(10). Another study in Asia revealed that the proportion of cesarean section In India $21.6 \%(11)$, in Pakistan $21.40 \%$ and China $54.5 \%(12,13)$. The study conducted in the Arab region showed that Egypt having the highest CS rate (26.2\%) but lowest in Mauritania 5.3\%(14). Another study conducted in 34 Sub-Saharan Africa (SSA) countries showed that the rate of cesarean section is ranged from $3 \%$ in Burkina Faso to $15.6 \%$ in Ghana. The highest rate showed in Rwanda, Namibia, and Ethiopia which is $64.2 \%, 60.3 \%$ and $30.1 \%$ respectively The lowest rate was shown in Congo which is $2.3 \%(15)$. Another study conducted in South Africa, Johannesburg CS rate was $39.4 \%(16)$.

Studies in Ethiopia that was conducted in private and government hospitals in Harar town the overall prevalence of CS was 34.3\%(17). A hospital-based cross-sectional study done at Chiro Zonal Hospital, West Harergae rate of cesarean delivery was $18.2 \%(18)$. A cross-sectional retrospective study that was conducted in Attat Hospital; Gurage zone Southern Nations and Nationalities of people Republics (SNNPR), Ethiopia, showed that the rate of CS was 27.6\%(9). Similarly, the study that was done in Oromia suggested that prevalence of CS was $29.4 \%(19)$. A hospital-based retrospective cross-sectional study carried at Jimma University specialized hospital the overall prevalence of C/S was $28.1 \%(20)$. According to the study conducted at Adigrat hospital, northern Ethiopia using a retrospective study design, the institutional cesarean delivery rate was $14.23 \%(21)$. The study that was done in the west Tigray zone, the rate of CS was $13.2 \%(22)$. According to the study conducted in Addis Ababa on cesarean delivery practices in teaching public and nongovernment/private maternal and child health $(\mathrm{MCH})$ hospitals, the CSR $31.1 \%$ in public hospitals and $48.3 \%$ in private hospitals(23). Similar Studies conducted in northwest Ethiopia the prevalence of CS was $11 \%$ in Finoteselam hospital and $25.4 \%$ in felegehiwot hospital(24, 25).

Study finding in India showed that Previous lour segment cesarean sections (LSCS) was the leading indication to the CS rate $(29.96 \%)$ followed by the arrest of labor $(13.94 \%)$, cephalio public disproportions(CPD) (11.84\%) and fetal distress (10.97\%)(26). A study conducted in Pakistan showed that the most common indication of cesarean section was the previous cesarean section scar $(22.76 \%)$ followed by failed progress of labor (18.29\%)(12). Another study conducted in Iran showed that repeated cesarean section (52.9\%), elective CS (on maternal request) (7.5\%) was the most common indication(27).

Similar Study conducted in Nigeria the common indication for CS was CPD $(40 \%$ followed by preeclampsia(18\%) and previous CS(11.8\%)(28). Another study that was conducted in Tanzania showed that prolonged/obstructed labor count (30\%) of all indication (29). Similarly study in Zambia suggested that fetal distress (14.9\%) and prolonged labor (10\%) was the commonest indication for CS(30).

Even though the cesarean section is important and lifesaving intervention for mothers and babies when vaginal delivery is contraindicated, it has a negative health impact on mother and baby and also put the 
mother on risk for future pregnancy. Therefore in order to decrease this negative health impact, the cesarean section rate should be in recommendation range. So identifying the prevalence and associated factor of the cesarean section would help our hospital and the country, in general, to set plan and strategies' to make cesarean section delivery rate within $5 \%-15 \%$ as WHO-recommended and to reduce maternal and fetal complication during delivery.

\section{Methods}

Study design and setting

An institution-based retrospective cross-sectional quantitative study has been conducted in the University of Gondar comprehensive referral hospital from March 1st to May 30th, 2019. University of Gondar compressive hospital is one of the oldest institutions in Ethiopia and producing the number of professionals and also giving huge service for the community since 1954.

Study Participants and Sampling Method

All laboring mothers admitting to the University of Gondar Referral Hospital were study population. The sample size was determined by using single population proportion formula based on the findings conducted in Bahirdar Felege Hiwot Referal Hospital which is $25.4 \%$, 4\% precision, $95 \%$ confidence level, $10 \%$ none response rate(25). With this assumptions sample of 323 laboring mothers were involved in the study.

Data Collection Procedure and Data Quality Control

The structured questionnaire prepared in English was translated to Amharic which is the local language by language experts and translated back to English by other language experts to check its consistency. Amharic version questionnaire was used to collect data. Three BSc holder midwives with minimum twoyear work experience were selected randomly and one other midwifery holder was selected for supervision.

The pre-test was done on 16 (5\% of sample size) on poly health canter found in Gondar town. Based on the finding grammatical sequences of questions were arranged on questionnaires. Training and orientation were given to the selected data collectors and supervisor for one day.

The Principal Investigator and Supervisor confirmed on the daily base the collected data for the completeness, accuracy, and clarity with data collectors, and the necessary correction was made before the next data collection day started. Finally, data cleaning; data entry, coding and crosschecking was done before data analysis.

Data quality assurance and analysis 
Data were entered to EPI info version 7 and imported to SPSS version 20 for analysis. Exploratory and statistical data analysis was done and results were presented using tables. Bivariate logistic regression was first fitted to identify potential confounding factors and variables with a p-value less than 0.2 were entered to multivariable logistic regression models using backward selection method to identify associated factors with knowledge of students on liberalized low of safe abortion. Adjusted odds ratio with $95 \%$ confidence interval and $p$-value $<0.05$ were used to show statistical significance with the outcome variable.

\section{Results}

A total of 323 study participants were included in this study with a response rate of $100 \%$.

The mean age of the study participants was 28.34 years $(S D= \pm 6.1)$. And more than half $(52 \%)$ of the study participant was in the age group of 25-34 years. Majority of the participants (94\%) were orthodox Christian followers. Nearly $97 \%$ of the women were Amhara in ethnicity. And also, the majority of participants 298 (92.3\%) were married. It was also noted that 178 women (55.1\%) lived in the urban area. As to the educational status of respondents, 95(29.4\%) were grade 9-12. The occupation of the respondents 203 (63.8\%) were housewives (Table 1).

\section{Obstetric characteristics}

Among the participants181 (56\%) of the women ever had the pregnancy of $2-4$. And nearly $41 \%$ of the participants gave birth after the seventh month one time. It was also noted that $86.7 \%$ of respondent have ANC follow up. Of the total participant's majority (86.7\%) of them have at least one ANC follow up, of these $48.3 \%$ women receives four ANC visit. More than $77 \%$ of the cesarean section ware performed between the gestational ages of 37-42 week (Table.1).

Indication of cesarean section

Of the total CS performed, the top five indications were NRFHP (17.8\%), previous CS scar (15.9\%), severe preeclampsia (12.1\%), abnormal fetal presentation (8.4\%), and failed induction (6.5\%) (Table 1).

The proportion of Cesarean section

The proportion of cesarean section rate was $29.7 \%$ (Cl: $24.2,36.2)$. From these more than $67 \%$ of women have an emergency cesarean section and around $80.2 \%$ of women have a primary cesarean section

(figure 1).

Factors associated with cesarean section

Bivariable and multivariable logistic regression was fitted to identify factors associated with cesarean section. On bivariable logistic regression, the religion of the women, educational status, maternal occupation, gravidity, parity, ANC follow up and number of ANC follow up had an association with 
cesarean section. The result of multivariable logistic regression showed that educational status of the mother, gravidity; parity, ANC follow up and the number of ANC visit were significantly associated with cesarean section.

The odds having a cesarean section for those mothers who are grade $1-8$ is 2.9 times compared with their counterpart (AOR,2.897, Cl:1.278,6.564).

Those women who got pregnant two-four times are 3.26 times more likely to have a cesarean section rate compared with those who got pregnant five or more times (AOR $=3.26, \mathrm{Cl}: 1.48,7.16)$.

Those mothers who hadn't ANC follow up in their last pregnancy were $75.2 \%$ less likely to cesarean section compared with their contour part $(A O R=0.248, \mathrm{Cl} 0.083-0.739)$.

Among respondents, those mothers who had ANC follow up of three times were 5.17 times more likely to have a cesarean section compared with those mothers who have one ANC visit $(A O R=5.17, \mathrm{Cl} 1.48$ 18.00).

Similarly, those women who had ANC visit of four were 4.49 times more likely to have cesareans section compared with mothers who had one ANC visit (AOR=4.49, $\mathrm{Cl}$ 1.47-13.66) ( Table 2).

\section{Discussion}

This study was aimed to assess the proportion and associated factors of cesarean section among mother who gave birth in the last three months in Gondar university comprehensive referral hospital.

This study found that the overall proportion of cesarean section is $29.7 \%$ (95\% Cl: $24.2 \%-36.2 \%$ ) and also $67.7 \%$ of the cesarean section was emergency and $80.2 \%$ were primary cesarean section.

This result is in line with a study conducted in Brazil and the Gurage zone which was $29.9 \%$ and $27.6 \%$ respectively $(9,10)$. Also, the study relatively comparable with studies conducted in Oromia $(29.4 \%)$, Jimma (28.1\%), felegehiwot hospital $(25.4 \%)(19,20,25)$. However, this finding is considerably high compared with research conducted in chiro zone hospital west Harergae, adigrat, and Tigray region which was $18.2 \%, 14.2 \%$, and $13.2 \%$ respectively $(18,21,22)$. This disparity might be due to the time gap, variation in sample size, increased awareness of mothers on ANC follow up and early identification of complications and increased opted cesarean section. Besides, this finding is significantly high compared with a study conducted in Finoteselam hospital $(11 \%)(24)$. This gross variation might be due to the time gap, variation in sample size, the difference in awareness and preference for CS also.

The major indication for cesarean section in the study was NRFHR $17.8 \%$ and previous cesarean section $15.9 \%$. This study was similar to the study conduct in Iran, chiro zona hospital, west Harergae, Addis Ababa nongovernmental $\mathrm{MCH}$ hospital $(18,23,27)$. However, this finding was different from the study conducted in Tigray that the most frequent indication was CPD (23.5\%)and ant partum hemorrhage (15.6\%) and also from study conduct in Nigeria the lading indication for cesarean were CPD $(40 \%$ 
followed by preeclampsia(18\%). This difference might be due to the decreased trial of vaginal birth after $\mathrm{CS}$ and time not given for conservative management of fetal distress.

As this study demonstrated that the odds of having CS among women who got pregnant two-four times were 3.26 times more likely to have CS compared with their counterpart ( $A O R=3.26, \mathrm{Cl}: 1.48,7.16)$. This result is in accordance with a study done in Brazil(10).

This study also demonstrates that those mothers who had ANC follow up of three times were 5.17 times more likely to have cesarean section compared with those mothers who have one ANC visit $(A O R=5.17, \mathrm{Cl}$ 1.48-18.00). These findings were similar to the study conducted in Addis Ababa (AOR=1.8, $\mathrm{Cl}$ 1.01-3.29) (20). This might be that when women have more ANC visit were more risk is going to be identified than those who do not have more ANC visit,

\section{Conclusions}

Based on this finding it was concluded that the overall CS prevalence in the university of Gondar was $29.7 \%$. It is above $15 \%$ recommended by WHO for developing countries. CS rates higher than $15 \%$ are not associated with a reduction in maternal and newborn mortality rates. Therefore to reduce the high prevalence of CS, each case should be evaluated to determine the possibility for vaginal delivery. As a result, this study confirms that even though the cesarean section is the most commonly performed surgical procedures to day, it is not without risks. The result of this study agrees with the other authors that the proper diagnosis of maternal and feta indications and also the use of prophylactic antibiotics helps to reduce the morbidity associated with CS. In multivariate logistic regression factor identified to be significantly associated with CS are educational states, gravidity, ANC follow up and number of ANC follow up. The leading indication of C/S was NRFHRP and previous $\mathrm{c} / \mathrm{s}$ scare.

\section{Limitations}

Referral case might overestimate the true magnitude of CS rate

\section{Declarations}

Ethical approval and consent

Ethical clearance letter was obtained From the Ethical Review Committee of the School of Midwifery, under the delegation of the Institution Review board (IRB) of the University of Gondar. Permission letters were also obtained from the University of Gondar referral hospital administrative office. Informed written consent was obtained from respondents after a clear explanation of the purpose of the study. Confidentiality and anonymity were maintained. 
Consent for publication

Not applicable

Availability of data and materials

Data will be available upon responsible request from the corresponding author using "sabayneh7@gmail.com"

Competing interests

The authors declared no potential competing interest with respect to the research, authorship, and publication of this article.

Funding

The authors have declared that no financial support in the research, authorship, and publication of this article was received.

Author's contributions

AAS has contributed from the beginning of the research idea to proposal writing, data collection process, analysis and interpretation of finding and preparation of the manuscript.

Acknowledgments

My heartfelt thanks also are given for Gondar University referral hospital and data collectors for their contribution to accomplishing this thesis and I would like to acknowledge, University of Gondar College of Medicine and health sciences, School of midwifery for ethical approval to prepare this thesis.

Author's information

1AA is Lecturer, School of Midwifery, College of Medicine and Health Sciences, University of Gondar, Ethiopia. AA has BSc Midwifery, MSc in clinical Midwifery.

\section{References}

1. AQ. BCf.cesarean section rate in developing countries.

2. Betrán AP YJ, Moller A, Zhang J, Gülmezoglu AM. The Increasing Trend in Caesarean Section Rates: Global , Regional and National Estimates 1990-2014. 2016;1-12.

3. Syed U KN, Khan A, Wall S. Care-seeking practices in South Asia: using formative research to design program interventions to save newborn lives. 2008;9-13; 9-13.

4. Gibbons L BJ, Lauer JA, Betrán AP, Merialdi M, Althabe F. The Global Numbers and Costs of Additionally Needed and Unnecessary Caesarean Sections Performed per Year: Overuse as a Barrier 
to Universal Coverage. 2010.

5. Gregory K JS. Cesarean versus Vaginal Delivery: Whose Risks? Whose Benefits? Cesarean versus Vaginal Delivery: Whose Risks? Whose Bene fi ts? 2016;(August 2011).

6. S. G. Trend and socio-demographic differentials of Caesarean section rate in Addis Ababa, Ethiopia: analysis based on Ethiopia demographic and health surveys data. [Internet]Available from: Reproductive Health. 2014;11(1):1-6.

7. Neuman M AG, Azad K, Kuddus A, Osrin D, More NS, et al.; Prevalence and determinants of caesarean section in private and public health facilities in underserved South Asian communities: crosssectional analysis of data from Bangladesh, India and Nepal. 2014.

8. Daniel CN SS. Caesarean delivery: An experience from a tertiary institution in North Western Nigeria. 19(1):18-24.

9. AOF- M. Prevalence and Outcome of Caesarean Section in Attat Hospital, Gurage Zone, SNNPR, Ethiopia Abstract. iMedPub Journals. 2015;;7(4):4.

10. Vieira GO FL, Oliveira NF De, Silva LR, Vieira TDO. Factors associated with cesarean delivery in public and private hospitals in a city of northeastern Brazil: a cross-sectional study. 2015;1-9.

11. Shweta Yadav1* SK, Sachin Singh Yadav2, Bhumika Thakur3. Analysis of caesarean section rate, indications and complications: review from medical college Ambala, Haryana, India. International Journal of Reproduction, Contraception, Obstetrics and GynecologyYadav S et al Int J Reprod Contracept Obstet Gynecol. 2016 Oct;5(10).

12. Maimoona Hafeez AY, Nazia Badar, Muhammad Irfan Pasha, Nishat Akram, Bushra Gulzar. Prevalence and Indications of Caesarean Section in a Teaching Hospital. JIMSA. January-March 2014 Vol. 27 No. 1.

13. Xin Wang1 SH, Lei Hou1, Liying Zou1, Yan Ruan1 and Weiyuan Zhang. Caesarean deliveries in China. BMC Pregnancy and Childbirth. (2017) 17:54

14. M. Khawaja NCaRJ. Hospital-based caesarean section inthe Arab region: an overview. Eastern Mediterranean Health Journal. 2009; Vol. 15, № 2.

15. Sanni Yaya1* OAU, Agbessi Amouzou3 and Ghose Bishwajit1. Disparities in caesarean section prevalenceand determinants across sub-Saharan Africacountries. (2018) Global Health Research and Policy;3:19

16. Ayob DR. An Observation of the Caesarean SectionRate at a Teaching Hospital in Johannesburg. 2015.

17. Tsega F MB DY, MengeshaMM Prevalence of Caesarean Section in Urban Health Facilities and Associated Factors in Eastern Ethiopia: Hospital Based Cross Sectional Stud. J Preg Child Health $2015 ; 2$.

18. S. W. associated factors of caesarean delivery at ChiroZonal Hospital West HarergaeOromia Regional State. 2016. 
19. Alemayehu Abegizer1 GTF, Abdisa Gurmessa3. Operative Deliveries: Indications and Post operativeComplications at Mattu Karl Hospital, Oromia Regional State,south west Ethiopia. Journal of Health, Medicine and Nursing, 2015;Vol.16.

20. Yuya ATaM. One Year Retrospective Analysis of Prevalence of Caesarean Section in Jimma University Specialized Hospital, South Western Ethiopia. J Preg Child Health 2015;; 2:4.

21. Samson Kahsay1 GB, Alem Gebremariam3* and Betel Birhane. Determinants of Caesarean Deliveries and its Major Indications in AdigratHospital, Northern Ethiopia: A Case Control Studyepidemiology (sunnyvale), 2015;5:3.

22. Solomon Gebre1* ANaAH. Criteria Based Clinical Audit of Cesarean Section in a General Hospital inWest Tigray, Ethiopia. Journal of Women's Health Care. 2017, 6,6.

23. Aman H NS YL. Caesarean delivery practices in teaching public and nongovernment/private $\mathrm{MCH}$ hospitals,addis abeba. J Health .2014.

24. By Melaku KindieYenit TG, Mulat Adefires \& Atsede Mazengia Shiferaw. Cesarean Section Rate, Maternal and Fetal Outcome of Birth FollowingCesarean Section at Finoteselam Hospital, Northwest Ethiopia: ADescriptive Retrospective Dat. Global Journals Inc (USA). Year 2016;Volume 16 Issue 3 Version 1.0.

25. Fantu Eyowas Abebe1* AWG, Ashebir Negasi Kidane1 and Gizached Aynalem Eyassu. Factors leading to cesarean section deliveryat Felegehiwot referral hospital, NorthwestEthiopia: a retrospective record reviewReproductive Health (2016) 13:6

26. Reena Sharma* PD. Indications and rate of caesarean delivery at tertiary care hospital: aretrospective study. Int J Reprod Contracept Obstet Gynecol. 2017 Oct;6(10).

27. RezaieSardari B TF KM, Sheikh Fathollahi M, Manshori A..A survey on causes of caesarean sections performed at the university hospitals of Niknafs and AlilbnAbiTalibof Rafsanjan, Iran, in the second trimeste. JOHE. 2014;3(2).

28. Sule H.M.1 HZI, Shambe I.H.3. Caesarean Section Rate And its Indications in An Urban PrivateHospital in Jos, North Central Nigeria: A 15-Year Survey. Journal of Dental and Medical Sciences. I (June. 2017),; Volume 16, Issue 6 Ver. V.

29. Stokke LBaS. INDICATIONS FOR CESAREAN SECTION IN ST. JOSEPH MEDICAL HOSPITAL MOSHI, TANZANIA 2013.

30. Edwin Nkhata1* DM, Matthias Tembo2,Seter Siziya. The prevalence and indication for caesarean section at Ndola Central Hospital, Ndola,Zambia. J Health Sci. , 2016; ;3 (3):315-320.

\section{Tables}

Table1: Sociodemographic, obstetric characteristics and indications of cesarean section among women who gave birth in university of Gondar comprehensive referral hospital, North West Ethiopia, 2019(n=323) 


\begin{tabular}{lllll}
\hline Variable & & & Frequency & Percentage (\%) \\
\hline Age & & $\mathrm{n}=323$ & & \\
& $15-19$ & & 16 & 5 \\
& $20-24$ & 71 & 22 \\
& $25-34$ & 170 & 52.6 \\
$>35$ & & 66 & 20.4 \\
Religion & & $\mathrm{n}=323$ & & \\
& Orthodox & & 248 & 76.7 \\
& Muslim & & 57 & 17.6 \\
& Protestant & & 18 & 5.7 \\
& & & \\
Ethnicity & & & \\
& & & & \\
& Amhara & & 315 & 97.5 \\
& Tigray & & 1 & 2.2 \\
& Oromo & & 0.3
\end{tabular}

Marital status $\quad \mathrm{n}=323$

Married

298

92.3

divorced

11

3.4

single

$\begin{array}{ll}6 & 1.9\end{array}$

windowed

$8 \quad 2.4$

Residential area

$\mathrm{n}=323$

Urban

178

55.1

Rural

145

44.9

Maternal

$\mathrm{n}=323$

occupational status

House wife

206

63.8

Governmental employ

59

18.3

Farmer

25

7.7

Private employ

9

2.8

Merchant

20

6.2

Student

4

1.2

Educational status

$\mathrm{n}=323$

Unable to read and write

86

26.6

Abel to read and write

77

23.8

Grade 1-8

65

20.2

Grade9-12, diploma and above

95

29.4

Gravid

$n=323$ 


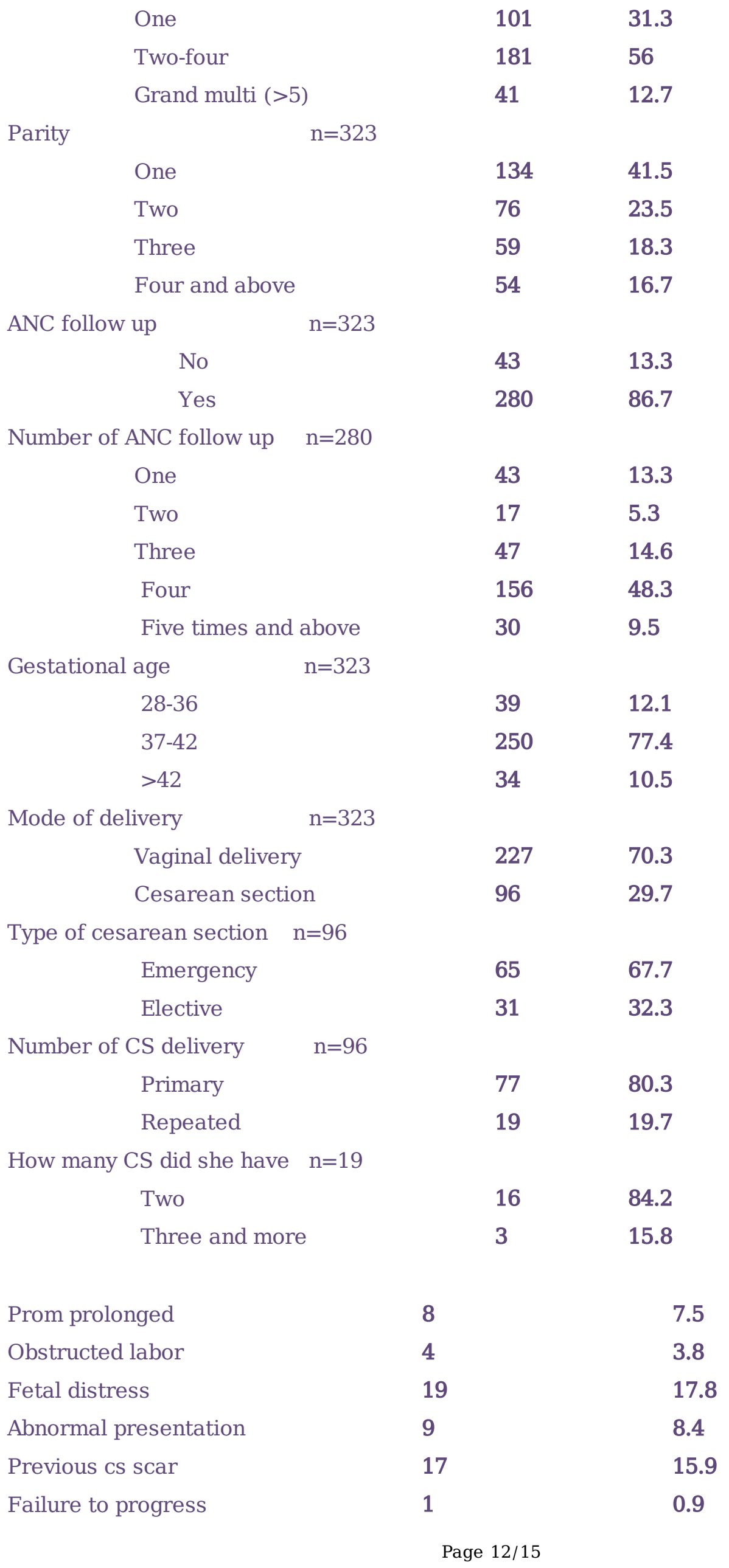




\begin{tabular}{lll} 
APH/AP/PP & 3 & 2.8 \\
Failed induction & 7 & 6.5 \\
Sever preeclampsia & 13 & 12.1 \\
Post date & 1 & 0.9 \\
Cord prolapsed & 2 & 1.9 \\
Multiple pregnancy & 4 & 3.7 \\
Maternal request & 1 & 0.9 \\
CPD & 6 & 5.9 \\
Macrosomia & 2 & 1.9 \\
Oligohydramnios & 3 & 2.8 \\
Pathological solid fibroid & 1 & 0.9 \\
Uterine rupture & 2 & 1.9 \\
Kidney disease & 1 & 0.9 \\
Contracted pelvis & 1 & 0.9 \\
\hline
\end{tabular}

Table 2: Factors associated with caesarean section delivery in bivariable and multivariable logistic regression among women who gave birth in university of Gondar comprehensive referral hospital North West Ethiopia, 2019. $(n=323)$ 


\begin{tabular}{|c|c|c|c|c|}
\hline \multirow[t]{2}{*}{ Variable } & Caesarean & section & \multirow[t]{2}{*}{ COR(95\% CI) } & \multirow[t]{2}{*}{ AOR(95\% CI) } \\
\hline & Yes\% & No\% & & \\
\hline \multicolumn{5}{|l|}{ Religion } \\
\hline Orthodox & $80(32.3)$ & $168(67.7)$ & $0.420(0118,1.492)$ & $0.361(0.91,1.430)$ \\
\hline Muslim & $13(22.8)$ & $44(77.2)$ & $0.677(0.169,2.705)$ & $0.443(0.98,2.009)$ \\
\hline Protestant & $3(16.7)$ & $15(83.3$ & 1 & 1 \\
\hline \multicolumn{5}{|l|}{ Occupation status } \\
\hline House wife & $64(31.1)$ & $142(68.9)$ & 1 & 1 \\
\hline Farmer & $13(22.0)$ & $46(78.0)$ & $1.595(0.806,3.156)$ & $2.918(1.122,7.587)$ \\
\hline Government employ & $9(36.0)$ & $16(64)$ & $0.801(0.336,1.909)$ & $0.882(0.339,2.292)$ \\
\hline Privet employs & $1(11.1)$ & $8(88.9)$ & $3.606(0.442,29.435)$ & $4.055(0.439,37.414)$ \\
\hline Merchant & $8(40.0)$ & $12(60)$ & $0.676(0.264,1.734)$ & $0.440(0.114,1.349)$ \\
\hline Student & $1(25.0)$ & $3(75)$ & $1.352(0.138,13.250)$ & $3.180(0.260,38.847)$ \\
\hline \multicolumn{5}{|l|}{ Educational status } \\
\hline Unable to read & $34(39.5)$ & $52(60.5)$ & 1 & 1 \\
\hline Abel to read & $19(24.7)$ & $58(75.3)$ & 1.996(1.017,3.919) & $2.459(1.165,5.192)$ \\
\hline Grad 1-8 & $12(18.5)$ & $53(81.5)$ & $2.888(1.349,6.183)$ & $2.897(1.278,6.56) *$ \\
\hline Grad 9 and above & $31(32.6)$ & $64(67.4)$ & $1.350(0.734,2.482)$ & $1.678(0.820,3.437)$ \\
\hline \multicolumn{5}{|l|}{ Gravidity } \\
\hline One & $38(37.6)$ & $63(62.4)$ & $1.432(0.687,2.983)$ & $1.429(0.620,3.296)$ \\
\hline Two-four & $39(21.5)$ & $142(78.5)$ & $3.145(1.548,6.388)$ & $3.259(1.484,7.160)^{*}$ \\
\hline Grand multi & $19(46.3)$ & $22(53.7)$ & 1 & 1 \\
\hline \multicolumn{5}{|l|}{ Parity } \\
\hline One & $43(32.1)$ & $91(67.9)$ & $1.347(0.699,2.596)$ & $1.071(0.236,4.857)$ \\
\hline Two & $20(26.3)$ & $56(73.7)$ & $1.782(0.8843,3.766)$ & $0.734(0.218,2.473)$ \\
\hline Three & $12(20.3)$ & $47(29.7)$ & $2.492(1.079,5.758)$ & $0.975(0.290,3.275)$ \\
\hline Four and above & $21(38.9)$ & $33(61.1)$ & 1 & 1 \\
\hline \multicolumn{5}{|l|}{ ANC follow up } \\
\hline Yes & 87(31.1) & 193(68.9) & 1 & 1 \\
\hline No & $9(20.9)$ & $34(79.1)$ & $0.587(0.270,1.277)$ & $0.248(0.083,0.739){ }^{*}$ \\
\hline \multicolumn{5}{|c|}{ Number of ANC follow up } \\
\hline One & $4(9.3)$ & $39(90.7)$ & 1 & 1 \\
\hline Two & $5(29.4)$ & $12(70.6)$ & $3.306(1.184,9.229)$ & $2.405(0.607,9.522)$ \\
\hline Three & $10(21.3)$ & $37(28.7)$ & $2.844(0.752,10.758)$ & $5.167(1.481,18.03)^{*}$ \\
\hline Four & $54(34.0)$ & $105(66.0)$ & $4.375(1.320,14.504)$ & $4.485(1.422,13.66)^{*}$ \\
\hline Five times and above & $14(46.7)$ & 16(53.3) & $3.237(1.190,8.811)$ & $1.755(0.767,4.017)$ \\
\hline
\end{tabular}

Hint* $=$ p-value $\leq 0.05$ 
Figures

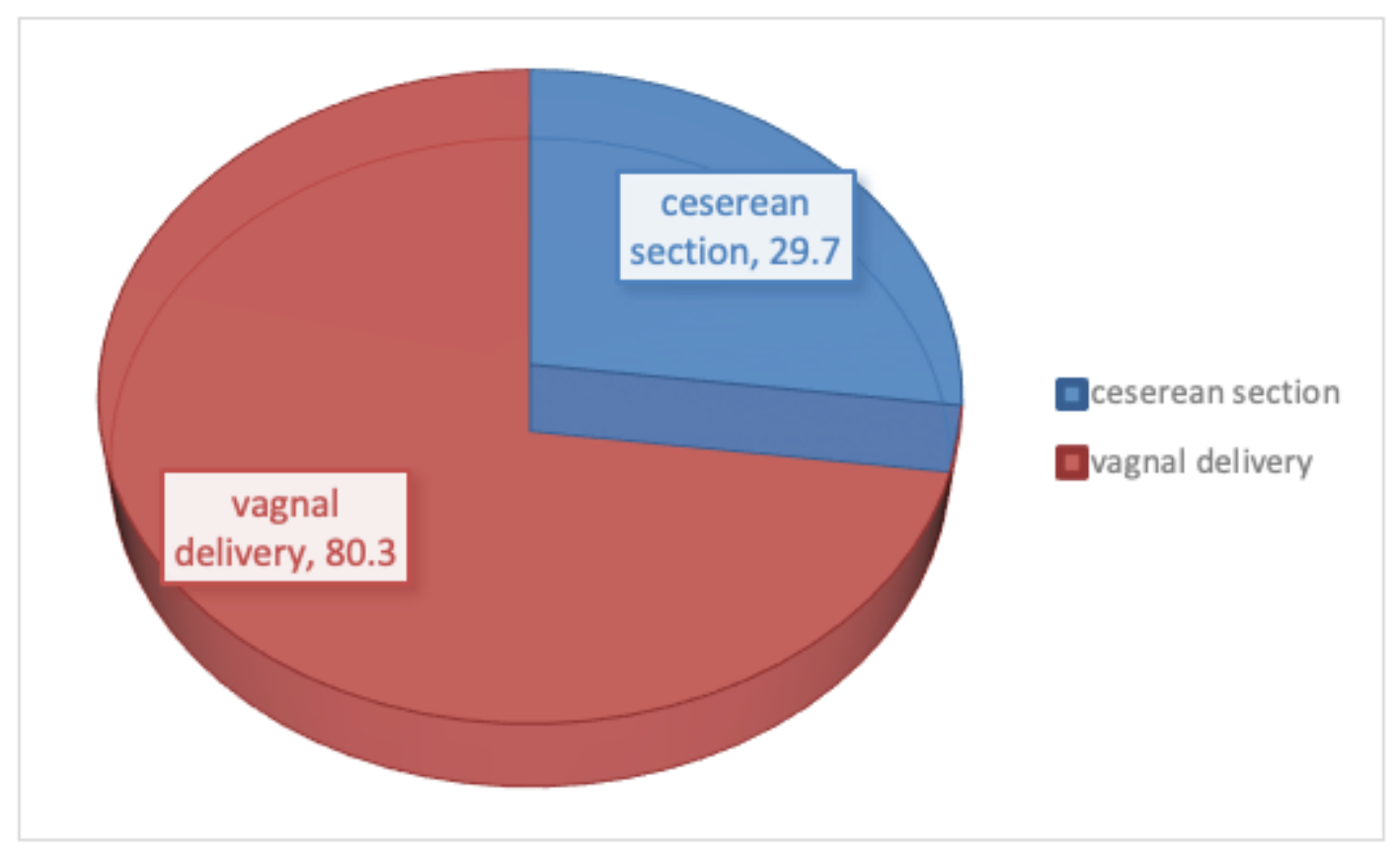

\section{Figure 1}

Pie chart showing cesarean section rate among women who give birth in university of Gondar compressive referral hospital, North West Ethiopia, $2019(n=323)$ 